ITERnational Electronic Journal of Algebra

VOLUMe 23 (2018) 131-142

DOI: $10.24330 /$ ieja.373654

\title{
GORENSTEIN HOMOLOGICAL DIMENSIONS WITH RESPECT TO A SEMIDUALIZING MODULE
}

\author{
Zhen Zhang and Jiaqun Wei \\ Received: 22 March 2017; Revised: 8 August 2017; Accepted: 8 August 2017 \\ Communicated by A. Çiğdem Özcan
}

\begin{abstract}
In this paper, let $R$ be a commutative ring and $C$ a semidualizing module. We investigate the (weak) $C$-Gorenstein global dimension of $R$ and we get a simple formula to compute the $C$-Gorenstein global dimension. Moreover, we compare it with the classical (weak) global dimension of $R$ and get the relations between them. At last, we compare the weak $C$-Gorenstein global dimension with the $C$-Gorenstein global dimension and we get that they are equal when $R$ is Noetherian.
\end{abstract}

Mathematics Subject Classification (2010): 13D02, 13D05, 13D07

Keywords: Semidualizing module, trivial extension ring, $C$-Gorenstein projective dimension

\section{Introduction}

The notion of semidualizing module was studied more than 27 years ago under other names by, e.g., Foxby [6] (PG-modules of rank 1), Golod [7] (suitable modules) and Vasconcelos [12] (spherical modules), which can be viewed as a generalization of dualizing module and free module of rank one. Relative algebra with respect to a semidualizing module has caught many authors' attention. Let $C$ be a semidualizing module over commutative Noetherian ring $R$, Holm and Jørgensen [9, Definition 2.7] introduced the notions of $C$-Gorenstein projective (injective and flat) modules, which are build from projective (injective and flat) and $C$-projective (injective and flat) modules, respectively. White [14] defined the $C$-Gorenstein projective (injective) modules over any commutative ring. In this field, projective (injective, flat) modules are generalized to $C$-projective (injective, flat) modules and Gorenstein projective (injective, flat) modules are generalized to $C$-Gorenstein projective (injective, flat) modules, etc., and the classical homological algebra is generalized to the Gorenstein homological algebra induced by a semidualizing module $C$. For this topic, we refer the reader to $[9,11,14]$.

This research was partially supported by the Shan Dong Provincial Natural Science Foundation of China (No.ZR2015PA001) and National Natural Science Foundation of China (No. 11371196). 
The homological dimension which arises by resolving a given module by $C$ Gorenstein projective (flat) or $C$-Gorenstein injective modules is known as the $C$-Gorenstein projective (flat) or $C$-Gorenstein injective dimension of the module. Bennis and Mahdou investigated the global Gorenstein dimension and weak global Gorenstein dimension of an associative ring $R$. They showed that the supremum of the Gorenstein projective dimensions of all the $R$-modules is equal to the supremum of the Gorenstein injective dimensions over an associative ring $R$ and that the supremum of the Gorenstein flat dimensions is smaller than the common value of the terms of this equality, cf. [2, Theorem 1.1]. It is natural to ask whether the global Gorenstein projective dimension with respect to a semidualizing module is equal to the global Gorenstein injective dimension of $R$. On the other hand, Holm and Jørgensen [9, Theorem 2.16] studied the trivial extension of $R$ by $C$, denoted by $R \ltimes C$. They showed that the $C$-Gorenstein projective, injective and flat $R$-module is in fact the Gorenstein projective, injective and flat $R \ltimes C$-module over commutative Noetherian ring $R$, respectively. However, their conclusions only applies to $R$-modules which are viewed as $R \ltimes C$-modules via the natural surjection $(R \ltimes C \rightarrow R)$. We are not sure whether they hold true for $R \ltimes C$-modules. Hence it is not trivial to show that the global Gorenstein projective dimension with respect to a semidualizing module $C$ of a ring $R$ is equal to the global Gorenstein injective dimension of $R$. In this paper, we use a new technique to show the global Gorenstein dimension induced by $C$ is definable. Obviously, it is not a trivial extension of [2, Theorem 1.1]. Moreover, we showed the following theorems over any commutative ring $R$.

Theorem. Let $C$-Ggldim $(R)$ denote the Gorenstein global dimension of $R$ induced by $C$. If $C$-Ggldim $(R)<\infty$, then $C-G g l d i m(R)=\sup \{C-G p d(R / I) \mid I$ is an ideal of $R\}$, where $C-G p d(R / I)$ is the $C$-Gorenstein projective dimension of $R / I$.

Compared with the classical global dimension of $R$, denoted by $\operatorname{gldim}(R)$, we get that $C$-Ggldim $(R) \leq \operatorname{gldim}(R)$ in general and when $\operatorname{gldim}(R)<\infty$, they are equal.

Enochs and Jenda [4, Proposition 10.3.2] proved that every finitely presented Gorenstein projective $R$-module is Gorenstein flat over a left and right coherent ring. In this paper, we get the $C$-Gorenstein projective $R$-module is $C$-Gorenstein flat $R$-module when $C$-Ggldim $(R)<\infty$. Moreover, we have:

Theorem. Let $C$-wGgldim $(R)$ denote the supremum of the $C$-Gorenstein flat dimension of all $R$-modules. We have $C$-w $\operatorname{Ggldim}(R) \leq C$-Ggldim $(R)$. If $R$ is Noetherian, they are equal. 
If we let $C=R$, we get $w \operatorname{Ggldim}(R)=\operatorname{Ggldim}(R)$ over Noetherian ring $R$, which extends [2, Corollary 1.2].

Throughout this paper, $R$ is a commutative ring and $\operatorname{Mod} R$ is the category of all $R$-modules.

\section{Preliminaries}

In this section, we recall a number of definitions, notions and results which will be used throughout the paper. For the definitions of Gorenstein projective (injective, flat) modules we refer the readers to see $[2,8]$.

Definition 2.1. [8, Page 171] Let $\mathscr{X}$ be a subcategory of $R$-modules and $M$ an $R$-module.

(1) A left $\mathscr{X}$-resolution of $M$ is an exact sequence $\mathbf{X}=\cdots \rightarrow X_{1} \rightarrow X_{0} \rightarrow$ $M \rightarrow 0$ with each $X_{i} \in \mathscr{X}$.

(2) A right $\mathscr{X}$-resolution of $M$ is an exact sequence $\mathbf{X}=0 \rightarrow M \rightarrow X^{0} \rightarrow$ $X^{1} \rightarrow \cdots$ with each $X^{i} \in \mathscr{X}$.

The $\mathscr{X}$-projective dimension of $M$ is the quantity

$$
\mathscr{X}-p d(M)=\inf \left\{\sup \left\{n \geq 0 \mid X_{n} \neq 0\right\} \mid \mathbf{X} \text { is a left } \mathscr{X} \text {-resolution of } M\right\} .
$$

The $\mathscr{X}$-injective dimension of $M$, denoted by $\mathscr{X}$-id( $M)$ is defined dually.

Particularly, $p d(M), i d(M)$, and $f d(M)$ is, respectively, the classical projective, injective, and flat dimension of $R$-module $M$. And we use $\operatorname{Gpd}(M), \operatorname{Gid}(M)$, and $G f d(M)$ to denote, respectively, the Gorenstein projective, injective, and flat dimension of $M$.

Definition 2.2. $[14,1.8]$ An $R$-module $C$ is called semidualizing if

(1) $C$ admits a degreewise finitely generated projective resolution;

(2) the natural homothety map $R \longrightarrow \operatorname{Hom}_{R}(C, C)$ is an isomorphism;

(3) $\operatorname{Ext}_{\bar{R}}^{\geq 1}(C, C)=0$.

Let $C$ be a semidualizing $R$-module. The class of $C$-projective (flat) $R$-modules, denoted by $\mathcal{F}_{C}\left(\mathcal{P}_{C}\right)$ and $C$-injective $R$-modules, denoted by $\mathcal{I}_{C}$, consists of modules which have the form $C \otimes_{R} F, F$ is projective (flat) $R$-modules and $\operatorname{Hom}_{R}(C, I), I$ is injective $R$-module, cf. [10, Definition 5.1].

By $C$-flat (projective, injective) $R$-modules, Holm and Jørgensen defined the $C$ Gorenstein flat, projective and injective modules in commutative ring $R$, which are clearly the generalization of Gorenstein flat, projective and injective modules. 
Note that White [14] extended the definition of $C$-Gorenstein projective modules to the non-Noetherian ring, where she called $G_{C}$-projective modules, we refer the reader to $[9,14]$.

Definition 2.3. [9, Definition 2.7] Let $C$ be a semidualizing $R$-module. An $R$ module $M$ is called $C$-Gorenstein injective if:

(1) $\operatorname{Ext}_{R}^{i>1}\left(\operatorname{Hom}_{R}(C, I), M\right)=0$ for all injective $R$-modules $I$.

(2) There exist injective $R$-modules $I_{0}, I_{1}, \cdots$ together with an exact sequence:

$$
\cdots \rightarrow \operatorname{Hom}_{R}\left(C, I_{1}\right) \rightarrow \operatorname{Hom}_{R}\left(C, I_{0}\right) \rightarrow M \rightarrow 0 .
$$

such that it stays exact when we apply the functor $\operatorname{Hom}_{R}\left(\operatorname{Hom}_{R}(C, J),-\right)$ for any injective $R$-module $J$.

$M$ is called $C$-Gorenstein projective if:

(1) $\operatorname{Ext}_{R}^{i \geq 1}\left(M, C \otimes_{R} Q\right)=0$ for all projective $R$-modules $Q$.

(2) There exist projective $R$-modules $Q_{0}, Q_{1}, \cdots$ together with an exact sequence:

$$
0 \rightarrow M \rightarrow C \otimes_{R} Q_{0} \rightarrow C \otimes_{R} Q_{1} \rightarrow \cdots .
$$

such that it stays exact when we apply the functor $\operatorname{Hom}_{R}\left(-, C \otimes_{R} Q\right)$ for any projective $R$-module $Q$.

$M$ is called $C$-Gorenstein flat if:

(1) $\operatorname{Tor}_{i \geq 1}^{R}\left(\operatorname{Hom}_{R}(C, I), M\right)=0$ for all injective $R$-modules $I$.

(2) There exist flat $R$-modules $F^{0}, F^{1}, \cdots$ together with an exact sequence:

$$
0 \rightarrow M \rightarrow C \otimes_{R} F^{0} \rightarrow C \otimes_{R} F^{1} \rightarrow \cdots,
$$

such that it stays exact when we apply the functor $\operatorname{Hom}_{R}(C, I) \otimes_{R}-$ for any injective $R$-module $I$.

Remark 2.4. By [9, Example 2.8], projective modules are $C$-Gorenstein projective, injective modules are $C$-Gorenstein injective and flat modules are $C$-Gorenstein flat over commutative Noetherian ring $R$. However, the condition of $R$ being Noetherian is not needed, which can be easily seen from the proof process in [9]. Hence every $R$-module $M$ admits $C$-Gorenstein projective (injective and flat) resolution and the $C$-Gorenstein projective (injective, flat) dimension of the $R$-module $M$ is definable over any commutative ring $R$.

By [9, Definition 9], let $C-G p d(M), C-G i d(M)$ and $C-G f d(M)$, denote the $C$ Gorenstein projective, injective and flat dimension of $M$, respectively.

At last, we recall the definition of trivial extension:

Definition 2.5. Let $R$ be a ring and $C$ a semidualizing module. The direct sum $R \oplus C$ can be equipped with the product: 


$$
(r, c) \cdot\left(r^{\prime}, c^{\prime}\right)=\left(r r^{\prime}, r c^{\prime}+r^{\prime} c\right) .
$$

This turns $R \oplus C$ into a ring which is called the trivial extension of $R$ by $C$ and denoted by $R \ltimes C$.

There are canonical ring homomorphisms, $R \rightleftarrows R \ltimes C$, which enable us to view $R$-modules as $R \ltimes C$-modules, and vice versa.

\section{Gorenstein global dimensions induced by $C$}

In this section, we investigate the (weak) $C$-Gorenstein global dimension of $R$. Firstly, we prove an important lemma, which makes [9, Theorem 2.16(1),(2)] hold true over any commutative ring, not necessarily Noetherian ring. Hence we can show our main theorems over any commutative ring.

Lemma 3.1. Let $R$ be any commutative ring. For any $R$-module $M$ and integer $n$, we have:

(1) $\operatorname{Ext}_{R \ltimes C}^{n}\left(\operatorname{Hom}_{R}(R \ltimes C, E), M\right) \cong \operatorname{Ext}_{R}^{n}\left(\operatorname{Hom}_{R}(C, E), M\right)$, where $E$ is any injective $R$-module;

(2) $\operatorname{Ext}_{R \ltimes C}^{n}\left(M,(R \ltimes C) \otimes_{R} Q\right) \cong \operatorname{Ext}_{R}^{n}\left(M, C \otimes_{R} Q\right)$, where $Q$ is any projective $R$-module.

Proof. We only prove (1) and the proof of (2) is similar.

By Definition 2.5, there exists an $R$-module isomorphism $R \ltimes C \cong R \oplus C$. So $\operatorname{Hom}_{R}(R \ltimes C, C) \cong R \ltimes C$ by Definition 2.2. Moreover, $C$ is a finitely presented $R$-module, so $R \ltimes C$ is also a finitely presented $R$-module. By [4, Theorem 3.2.11],

$\operatorname{Hom}_{R}(R \ltimes C, E) \cong \operatorname{Hom}_{R}\left(\operatorname{Hom}_{R}(R \ltimes C, C), E\right) \cong(R \ltimes C) \otimes_{R} \operatorname{Hom}_{R}(C, E)$.

Consider the projective resolution of the $R$-module $\operatorname{Hom}_{R}(C, E)$,

$$
\mathbb{P}=\cdots \rightarrow P_{1} \rightarrow P_{0} \rightarrow \operatorname{Hom}_{R}(C, E) \rightarrow 0 .
$$

By [10, Corollary 6.1], $\operatorname{Hom}_{R}(C, E) \in \mathcal{A}_{C}(R)$. So $\operatorname{Tor}_{i \geq 1}^{R}\left(R \ltimes C, \operatorname{Hom}_{R}(C, E)\right)=0$. Thus we get another exact sequence after applying the functor $(R \ltimes C) \otimes_{R}-$ to $\mathbb{P}$ :

$$
\cdots \rightarrow(R \ltimes C) \otimes_{R} P_{1} \rightarrow(R \ltimes C) \otimes_{R} P_{0} \rightarrow(R \ltimes C) \otimes_{R} \operatorname{Hom}_{R}(C, E) \rightarrow 0 .
$$

By [9, Lemma 1.5], $(R \ltimes C) \otimes_{R} P_{i}$ is a projective $R \ltimes C$-module for any $i \geq$ 0 . So the above exact sequence is a projective resolution of the $R \ltimes C$-module 
$(R \ltimes C) \otimes_{R} \operatorname{Hom}_{R}(C, E)$. Hence we have that:

$$
\begin{aligned}
& \operatorname{Ext}_{R \ltimes C}^{n}\left(\operatorname{Hom}_{R}(R \ltimes C, E), M\right) \\
\cong & \operatorname{Ext}_{R \ltimes C}^{n}\left((R \ltimes C) \otimes_{R} \operatorname{Hom}_{R}(C, E), M\right) \\
= & H_{-n} \operatorname{Hom}_{R \ltimes C}\left((R \ltimes C) \otimes_{R} \mathbb{P}, M\right) \\
\cong & H_{-n} \operatorname{Hom}_{R}(\mathbb{P}, M) \\
= & \operatorname{Ext}_{R}^{n}\left(\operatorname{Hom}_{R}(C, E), M\right),
\end{aligned}
$$

where the second isomorphism is a Hom-tensor adjointness.

Remark 3.2. By Lemma 3.1, we know [9, Proposition 2.13 and Theorem 2.16(1), (2)] hold true over any commutative ring $R$, which can be easily seen from the proof process in [9].

Now, we show the $C$-Gorenstein global dimension of $R$ is definable. And we use a different method from [2].

Lemma 3.3. Let $E$ be any injective and $Q$ any projective $R$-module. Then we have

(1) $i d_{R \ltimes C}\left((R \ltimes C) \otimes_{R} Q\right) \leq i d_{R}\left(C \otimes_{R} Q\right)$;

(2) $p d_{R \ltimes C}\left(\operatorname{Hom}_{R}(R \ltimes C, E)\right) \leq p d_{R}\left(\operatorname{Hom}_{R}(C, E)\right)$.

Proof. (1) Let $\mathbb{I}=0 \rightarrow C \otimes_{R} Q \rightarrow E_{0} \rightarrow E_{1} \rightarrow \cdots$ be an injective resolution of the $R$-module $C \otimes_{R} Q$. By [10, Corollary 6.1], $C \otimes_{R} Q \in \mathcal{B}_{C}(R)$. We can get $\operatorname{Ext}_{R}^{\geq 1}\left(R \ltimes C, C \otimes_{R} Q\right)=0$ by Definition 2.5 and [10, Definition 4.1]. Hence the sequence $\operatorname{Hom}_{R}(R \ltimes C, \mathbb{I})$, i.e.,

$$
0 \rightarrow \operatorname{Hom}_{R}\left(R \ltimes C, C \otimes_{R} Q\right) \rightarrow \operatorname{Hom}_{R}\left(R \ltimes C, E_{0}\right) \rightarrow \operatorname{Hom}_{R}\left(R \ltimes C, E_{1}\right) \rightarrow \cdots
$$

is exact. By [9, Lemma 1.4], $\operatorname{Hom}_{R}\left(R \ltimes C, E_{i}\right)$ is an injective $R \ltimes C$-module for each $i \geq 0$. So $\operatorname{Hom}_{R}(R \ltimes C, \mathbb{I})$ is an injective resolution of the $R \ltimes C$-module $\operatorname{Hom}_{R}\left(R \ltimes C, C \otimes{ }_{R} Q\right)$. Since $C$ is finitely presented and $\operatorname{Hom}_{R}(R \ltimes C, C) \cong R \ltimes C$, we have $\operatorname{Hom}_{R}\left(R \ltimes C, C \otimes_{R} Q\right) \cong \operatorname{Hom}_{R}(R \ltimes C, C) \otimes_{R} Q \cong(R \ltimes C) \otimes_{R} Q$ by [4, Theorem 3.2.14]. So

$$
i d_{R \ltimes C}\left((R \ltimes C) \otimes_{R} Q\right)=i d_{R \ltimes C}\left(\operatorname{Hom}_{R}\left(R \ltimes C, C \otimes_{R} Q\right)\right) \leq i d_{R}\left(C \otimes_{R} Q\right) .
$$

(2) Let $\mathbb{P}$ be a projective resolution of the $R$-module $\operatorname{Hom}_{R}(C, E)$. Following from the proof of Lemma 3.1, $(R \ltimes C) \otimes_{R} \mathbb{P}$ is a projective resolution of $R \ltimes C$ module $\operatorname{Hom}_{R}(R \ltimes C, E)$. So $p d_{R \ltimes C}\left(\operatorname{Hom}_{R}(R \ltimes C, E)\right) \leq p d_{R}\left(\operatorname{Hom}_{R}(C, E)\right)$.

Lemma 3.4. Let $E$ be an injective and $Q$ a projective $R$-module. For any nonnegative integer $n$, we have

(1) if $C-G p d(E) \leq n$, then $\mathcal{P}_{C}-p d(E)=C-G p d(E)$;

(2) if $C-G i d(Q) \leq n$, then $\mathcal{I}_{C}-i d(Q)=C-G i d(Q)$. 
Proof. We only prove (1) and the proof of (2) is similar.

Clearly, $C-G p d(E) \leq \mathcal{P}_{C}-p d(E)$. We only need to show the inverse equality. Since $C-G p d(E) \leq n$, there exists an exact sequence by [14, Theorem 3.6]: $0 \rightarrow K \rightarrow G \rightarrow E \rightarrow 0$, where $\mathcal{P}_{C}-p d(K) \leq C$ - $G p d(E)-1$ and $G$ is $C$-Gorenstein projective. Note that $C$-Gorenstein projective dimensions are called $G_{C}$-projective dimension, denoted by $G_{C}-p d_{R} M$ in [14]. By the definition of $C$-Gorenstein projective module, we have the following push-out diagram

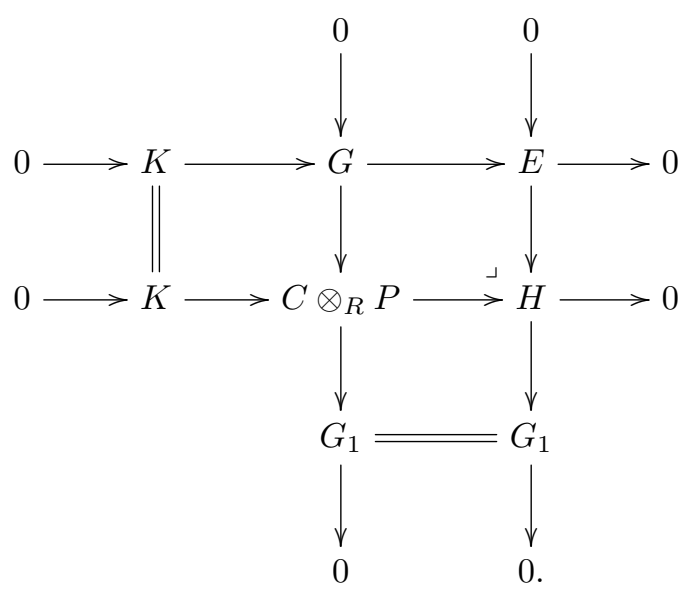

We deduce $\mathcal{P}_{C}-p d(H) \leq C-G p d(E)$ by the middle row in the push-out diagram. Since $E$ is injective, the exact sequence $0 \rightarrow E \rightarrow H \rightarrow G_{1} \rightarrow 0$ splits. So $E$ is a direct summand of $H$ and thus $\mathcal{P}_{C}-p d(E) \leq C-G p d(E)$. Hence the equality in (1) follows.

Proposition 3.5. For a non-negative integer n, if $\sup \{C-G p d(M) \mid M \in M o d R\} \leq$ $n$ (or $\sup \{C$-Gid $(M) \mid M \in M o d R\} \leq n)$, then for every projective $R \ltimes C$-module $P$ and injective $R \ltimes C$-module $I$, the following hold true.

(1) $i d_{R \ltimes C}(P) \leq n$;

(2) $p d_{R \ltimes C}(I) \leq n$.

Proof. By [9, Lemmas 1.4 and 1.5], we can easily know that any injective $R \ltimes C$ module $I$ is a summand in a module $\operatorname{Hom}_{R}(R \ltimes C, E)$ for some injective $R$-module $E$ and any projective $R \ltimes C$-module $P$ is a summand in a module $(R \ltimes C) \otimes_{R} Q$ for some projective $R$-module $Q$ over any ring $R$. So we only need to show

$$
i d_{R \ltimes C}\left((R \ltimes C) \otimes_{R} Q\right) \leq n \text { and } p d_{R \ltimes C}\left(\operatorname{Hom}_{R}(R \ltimes C, E)\right) \leq n .
$$

Firstly, we assume that $\sup \{C-G p d(M) \mid M \in M o d R\} \leq n$.

(1) Let $Q$ be any projective $R$-module. Then $\operatorname{Ext}_{R}^{i}\left(M, C \otimes_{R} Q\right)=0$ for any $R$-module $M$ and any $i>n$ by [14, Proposition 2.12]. Thus $i d_{R}\left(C \otimes_{R} Q\right) \leq n$. By Lemma 3.3(1), $i d_{R \ltimes C}\left((R \ltimes C) \otimes_{R} Q\right) \leq n$. 
(2) By Lemma 3.4(1), $\mathcal{P}_{C}-p d(E)=C$-Gpd(E) for any injective $R$-module $E$. So $\mathcal{P}_{C}-p d(E) \leq n$ by the assumption. By [10, Theorem 5.1], it is easy to show $p d\left(\operatorname{Hom}_{R}(C, E)\right) \leq n$. So $p d_{R \ltimes C}\left(\operatorname{Hom}_{R}(R \ltimes C, E)\right) \leq n$ by Lemma 3.3(2).

Now, we assume that $\sup \{C-G i d(M) \mid M \in M o d R\} \leq n$.

(1) By Lemma 3.4(2), $\mathcal{I}_{C^{-}}-i d(Q)=C$-Gid $(Q) \leq n$ for any projective $R$-module $Q$. So $i d_{R}\left(C \otimes_{R} Q\right) \leq n$ by [10, Theorem 5.1]. Hence $i d_{R \ltimes C}\left((R \ltimes C) \otimes_{R} Q\right) \leq n$ by Lemma $3.3(1)$.

(2) Let $M$ be any $R$-module, then $\operatorname{Ext}_{R}^{i}\left(\operatorname{Hom}_{R}(C, E), M\right)=0$ for all $i>n$ and all injective $R$-module $E$ by [9, Definition 2.7]. So $p d\left(\operatorname{Hom}_{R}(C, E)\right) \leq n$. Hence $p d_{R \ltimes C}\left(\operatorname{Hom}_{R}(R \ltimes C, E)\right) \leq n$ by Lemma 3.3(2).

Lemma 3.6. Let $n$ be a non-negative integer such that $i d(P) \leq n$ and $p d(E) \leq n$, where $P$ is any projective $R$-module and $E$ is any injective $R$-module. Then the following hold for any $R$-module $M$ :

(1) If $\operatorname{Gpd}_{R}(M)<\infty$, then $\operatorname{Gid}_{R}(M) \leq n$;

(2) If $\operatorname{Gid}_{R}(M)<\infty$, then $\operatorname{Gpd}_{R}(M) \leq n$.

Proof. It follows from [3, Theorem 4.1].

The following result was also proved in [15, Theorem 4.4]. However, we use a different method to prove it.

Proposition 3.7. Let $R$ be any commutative ring and $C$ a semidualizing $R$-module. Then

$$
\sup \{C-G i d(M) \mid M \in M o d R\}=\sup \{C-G p d(M) \mid M \in M o d R\} .
$$

Proof. We only show the inequality

$$
\sup \{C-G i d(M) \mid M \in M o d R\} \leq \sup \{C-G p d(M) \mid M \in M o d R\}
$$

and the proof of the reverse inequality is similar.

Suppose that $\sup \{C-G p d(M) \mid M \in M o d R\} \leq n$ for some non-negative integer $n$. Then for every projective $R \ltimes C$-module $P$ and injective $R \ltimes C$-module $I$, we have that $i d_{R \ltimes C}(P) \leq n$ and $p d_{R \ltimes C}(I) \leq n$ by Proposition 3.5. Moreover, let $M$ be any $R$-module, then $C-G p d(M) \leq n$. Thus $G p d_{R \ltimes C}(M) \leq n<\infty$ by $[9$, Theorem 2.16(1)] and Remark 3.2. So $\operatorname{Gid}_{R \ltimes C}(M) \leq n$ by Lemma 3.6. By [9, Theorem 2.16(2)] and Remark 3.2, C-Gid(M) $\leq n$. Hence $\sup \{C-G i d(M) \mid M \in$ $\operatorname{ModR}\} \leq n$ and the inequality holds true.

We call the common value in Proposition 3.7 $C$-Gorenstein global dimension of the ring $R$ and denote it by $C$-Ggldim $(R)$. It is easy to see that $C$-Gorenstein global dimension extends Gorenstein global dimension and global dimension of $R$. 
In the classical homological algebra, the global dimension of a ring $R$, denoted by $\operatorname{gldim}(R)$, can be computed via the following formula:

$$
\operatorname{gldim}(R)=\sup \{p d(R / I) \mid I \text { is an ideal of } R\} .
$$

We will show the $C$-Gorenstein global dimension of $R$ can also be computed via a similar formula.

Lemma 3.8. Let $R$ be a commutative ring with $C$-Ggldim $(R)<\infty$. Denoted by Proj $R$ the class of projective $R$-modules, we have

$$
C-\operatorname{Ggldim}(R)=\sup \left\{i d_{R}\left(C \otimes_{R} Q\right) \mid Q \in \operatorname{Proj} R\right\} .
$$

Proof. Assume that $\sup \left\{i d_{R}\left(C \otimes_{R} Q\right) \mid Q \in \operatorname{Proj} R\right\}=n$ for some non-negative integer $n$, then $i d_{R}(C \otimes Q) \leq n$ for any projective $R$-module $Q$. For any $R$-module $M$ and $i>n$, we have $\operatorname{Ext}_{R}^{i}\left(M, C \otimes_{R} Q\right)=0$. Also $C-G p d_{R} M<\infty$ by the assumption, thus $C-G p d_{R} M \leq n$ by [14, Proposition 2.12]. By Proposition 3.7, we get $C-\operatorname{Ggldim}(R)=\sup \left\{C-G p d_{R} M \mid M \in M o d R\right\} \leq n$.

On the other hand, assume that $C-\operatorname{Ggldim}(R)=n$ and $Q$ is any projective $R$-module. Then $\operatorname{Ext}_{R}^{i}\left(M, C \otimes_{R} Q\right)=0$ for any $R$-module $M$ and $i>n$ by [14, Proposition 2.12] and Proposition 3.7. Hence $i d_{R}\left(C \otimes_{R} Q\right) \leq n$. So

$$
\sup \left\{i d_{R}\left(C \otimes_{R} Q\right) \mid Q \in \operatorname{Proj} R\right\} \leq C-\operatorname{Ggldim}(R) .
$$

Theorem 3.9. If $C$-Ggldim $(R)<\infty$, then

$$
C-\operatorname{Ggldim}(R)=\sup \{C-G p d(R / I) \mid I \text { is an ideal of } R\} \text {. }
$$

Proof. It is clear that $\sup \{C-G p d(R / I) \mid I$ is an ideal of $R\} \leq C$ - $\operatorname{Ggldim}(R)$. Let $\sup \{C-G p d(R / I) \mid I$ is an ideal of $R\}=n<\infty$. By [14, Proposition 2.12], $\operatorname{Ext}_{R}^{n+1}\left(R / I, C \otimes_{R} Q\right)=0$ for every $R$-ideal $I$ and projective $R$-module $Q$. Consider the injective resolution of $C \otimes_{R} Q$,

$$
0 \rightarrow C \otimes_{R} Q \rightarrow E_{0} \rightarrow \cdots \rightarrow E_{n-1} \rightarrow T^{\prime} \rightarrow 0 .
$$

Applying $\operatorname{Hom}_{R}(R / I,-)$, we get that $\operatorname{Ext}_{R}^{1}\left(R / I, T^{\prime}\right) \cong \operatorname{Ext}_{R}^{n+1}\left(R / I, C \otimes_{R} Q\right)=0$. By [13, Theorem 9.11], $T^{\prime}$ is injective. So $i d_{R}\left(C \otimes_{R} Q\right) \leq n$. By Lemma 3.8, $C$ $\operatorname{Ggldim}(R) \leq n$. Therefore, $C-\operatorname{Ggldim}(R)=\sup \{C-G p d(R / I) \mid I$ is an ideal of $R\}$.

Remark 3.10. By Remark 2.4, C-Ggldim $(R) \leq \operatorname{gldim}(R)$. On the other hand, it is easy to show $\sup \left\{\mathcal{P}_{C}-p d(M) \mid M \in \operatorname{ModR}\right\}=\sup \{p d(N) \mid N \in \operatorname{ModR}\}=$ gldimR. So if $\operatorname{gldim}(R)<\infty$, then $\mathcal{P}_{C}-p d(M)<\infty$ for any $R$-module $M$. Hence $\mathcal{P}_{C}-p d(M)=C-G p d(M)$ by [14, Proposition 2.16]. So if $\operatorname{gldim}(R)<\infty$, then $\sup \left\{\mathcal{P}_{C}-p d(M) \mid M \in M o d R\right\}=\sup \{C-G p d(M) \mid M \in M o d R\}$, i.e., $C$ $\operatorname{Ggldim}(R)=\operatorname{gldim}(R)$. 
Now, we consider the the weak $C$-Gorenstein global dimension and denote it by $C$-w $\operatorname{Ggldim}(R)$,

$$
C \text {-w } \operatorname{Ggldim}(R)=\sup \left\{C-G f d_{R}(M) \mid M \in M o d R\right\} .
$$

Obviously, it is a generalization of weak Gorenstein global dimension and weak global dimension of $R$. By Remark 2.4, any flat $R$-module is $C$-Gorenstein flat. So $C$-wGgldim $(R) \leq w g l d i m(R)$, where $\operatorname{wgldim}(R)$ denotes the supremum of the flat dimensions of all the $R$-modules.

Enochs and Jenda [4] proved that every finitely presented Gorenstein projective $R$-module is Gorenstein flat over a left and right coherent ring. The following proposition indicates the $C$-Gorenstein projective $R$-module is $C$-Gorenstein flat when $C$-Ggldim $(R)<\infty$.

Proposition 3.11. Let $R$ be any ring with $C$-Ggldim $(R)<\infty$, then any $C$ Gorenstein projective $R$-module is $C$-Gorenstein flat.

Proof. Assume that $C$-Ggldim $(R) \leq n$ for some non-negative integer $n$. Then $C$-Gid $(N) \leq n$ for every $R$-module $N$. Hence $\operatorname{Ext}_{R}^{i>n}\left(\operatorname{Hom}_{R}(C, E), N\right)=0$ for any injective $R$-module $E$. So $p d\left(\operatorname{Hom}_{R}(C, E)\right) \leq n$ and $f d\left(\operatorname{Hom}_{R}(C, E)\right) \leq n$. Denote the character module of $\operatorname{Hom}_{R}(C, E)$ by $\operatorname{Hom}_{R}(C, E)^{+}$, then $i d\left(\operatorname{Hom}_{R}(C, E)^{+}\right) \leq$ $n$ by [4, Theorem 3.2.9]. So $\operatorname{Ext}_{\bar{R}}^{\geq 1}\left(C, \operatorname{Hom}_{R}(C, E)^{+}\right)=0$ by [10, Corollaries 6.1 and 6.2]. And $\operatorname{Ext}_{\bar{R}}^{\geq 1}\left(C \otimes P, \operatorname{Hom}_{R}(C, E)^{+}\right)=0$ by [13, Page 258, 9.20]. Let $M$ be a $C$-Gorenstein projective $R$-module, then there exists an exact sequence $\mathbb{P}=: 0 \rightarrow M \rightarrow C \otimes P^{0} \stackrel{f^{0}}{\rightarrow} C \otimes P^{1} \stackrel{f^{1}}{\rightarrow} \cdots$ with $P^{i}$ projective modules. Applying $\operatorname{Hom}\left(-, \operatorname{Hom}(C, E)^{+}\right)$to $\mathbb{P}$, by the dimension shifting argument, we get $\operatorname{Ext}^{i}\left(M, \operatorname{Hom}(C, E)^{+}\right) \cong E x t^{n+i}\left(k e r f^{n}, \operatorname{Hom}(C, E)^{+}\right)$for all $i \geq 1$. Since $i d\left(\operatorname{Hom}_{R}(C, E)^{+}\right) \leq n, \quad E x t^{\geq 1}\left(M, \operatorname{Hom}(C, E)^{+}\right)=0 . \quad$ By [4, Theorem 3.2.1], $\operatorname{Tor}_{\geq 1}^{R}(M, \operatorname{Hom}(C, E))=0$. From this, it is easy to see $\operatorname{Hom}\left(\mathbb{P}, \operatorname{Hom}(C, E)^{+}\right)$is exact. And the Hom-tensor adjointness

$$
\operatorname{Hom}_{\mathbb{Z}}(\operatorname{Hom}(C, E) \otimes \mathbb{P}, \mathbb{Q} / \mathbb{Z}) \cong \operatorname{Hom}\left(\mathbb{P}, \operatorname{Hom}(C, E)^{+}\right)
$$

implies that $\operatorname{Hom}(C, E) \otimes_{R} \mathbb{P}$ is exact. Hence $M$ is $C$-Gorenstein flat by Definition 2.3 .

To end this manuscript, we compare the $C$-Ggldim $(R)$ and $C$-wGgldim $(R)$ over any ring $R$.

Theorem 3.12. We always have $C$-wGgldim $(R) \leq C$-Ggldim $(R)$. If $R$ is Noetherian with $C$-Ggldim $(R)<\infty$, then $C$-w $\operatorname{Ggldim}(R)=C$-Ggldim $(R)$.

Proof. By Proposition 3.11, $C$-wGgldim $(R) \leq C$-Ggldim $(R)$.

When $R$ is Noetherian, we will show that $C$ - $\operatorname{Ggldim}(R) \leq C$-wGgldim $(R)$. In fact, suppose that $C$-w $\operatorname{Ggldim}(R)=n$ for some non-negative integer $n$, then for 
every finitely generated $R$-module $M, C-G f d(M) \leq n$. Consider the projective resolution of $M: 0 \rightarrow G_{n} \rightarrow P_{n-1} \rightarrow \cdots \rightarrow P_{1} \rightarrow P_{0} \rightarrow M \rightarrow 0$, with $P_{i}$ finitely generated projective for $0 \leq i \leq n-1$. Then $G_{n}$ is $C$-Gorenstein flat. Since $R$ is Noetherian and $M$ is finitely generated, so $G_{n}$ is finitely presented. By Definition 2.5, as $R \ltimes C$-module, $G_{n}$ is finitely generated. Since $R$ is Noetherian, $R \ltimes C$ is Noetherian by [5, Page 87]. So $G_{n}$ is a finitely presented Gorenstein flat $R \ltimes C$-module by [9, Theorem 2.16(3)] and thus $G_{n}$ is a Gorenstein projective $R \ltimes C$-module by [1, Proposition 1.3]. Hence $G_{n}$ is a $C$-Gorenstein projective $R$ module also by [9, Theorem 2.16(2)]. We get $C-G p d(M) \leq n$. Particularly,

$C$ - $\operatorname{Gpd}(R / I) \leq n$ for any $R$-ideal $I$. So $C$ - $\operatorname{Ggldim}(R) \leq n$ by Theorem 3.9. Hence $C$-Ggldim $(R) \leq C$-wGgldim $(R)$ and so $C$-w $\operatorname{Ggldim}(R)=C$-Ggldim $(R)$.

Remark 3.13. If $C=R$ in the above theorem, then $w \operatorname{Ggldim}(R)=\operatorname{Ggldim}(R)$ over Noetherian ring $R$, which extends [2, Corollary 1.2].

Acknowledgment. The authors would like to thank the referee for the valuable suggestions and comments.

\section{References}

[1] D. Bennis and N. Mahdou, Strongly Gorenstein projective, injective, and flat modules, J. Pure Appl. Algebra, 210(2) (2007), 437-445.

[2] D. Bennis and N. Mahdou, Global Gorenstein dimensions, Proc. Amer. Math. Soc., 138(2) (2010), 461-465.

[3] I. Emmanouil, On the finiteness of Gorenstein homological dimensions, J. Algebra, 372 (2012), 376-396.

[4] E. E. Enochs and O. M. G. Jenda, Relative Homological Algebra, De Gruyter Expositions in Mathematics, 3, Walter de Gruyter \& Co., Berlin, 2000.

[5] R. M. Fossum, P. A. Griffith and I. Reiten, Trivial Extensions of Abelian Categories, Homological algebra of trivial extensions of abelian categories with applications to ring theory, Lecture Notes in Mathematics, 456, Springer-Verlag, Berlin-New York, 1975.

[6] H.-B. Foxby, Gorenstein modules and related modules, Math. Scand., 31 (1972), 267-284.

[7] E. S. Golod, G-dimension and generalized perfect ideals, Trudy Mat. Inst. Steklov., 165 (1984), 62-66.

[8] H. Holm, Gorenstein homological dimensions, J. Pure Appl. Algebra, 189(1-3) (2004), 167-193.

[9] H. Holm and P. Jørgensen, Semi-dualizing modules and related Gorenstein homological dimensions, J. Pure Appl. Algebra, 205(2) (2006), 423-445. 
[10] H. Holm and D. White, Foxby equivalence over associative rings, J. Math. Kyoto Univ., 47(4) (2007), 781-808.

[11] S. Sather-Wagstaff, T. Sharif and D. White, Tate cohomology with respect to semidualizing modules, J. Algebra, 324(9) (2010), 2336-2368.

[12] W. V. Vasconcelos, Divisor Theory in Module Categories, North-Holland Mathematics Studies, 14, North-Holland Publishing Co., Amsterdam-Oxford; American Elsevier Publishing Co., Inc., New York, 1974.

[13] C. A. Weibel, An Introduction to Homological Algebra, Cambridge Stud. Adv. Math., 38, Cambridge University Press, Cambridge, 1994.

[14] D. White, Gorenstein projective dimension with respect to a semidualizing module, J. Commut. Algebra, 2(1) (2010), 111-137.

[15] G. Zhao and J. Sun, Global dimensions of rings with respect to a semidualizing module, avilable from https://arxiv.org/abs/1307.0628.

Zhen Zhang (Corresponding Author)

Institution of Mathematics

School of Mathematics Science

Nanjing Normal University

Nanjing 210023, China

(Department of Primary Education

ZiBo Normal College

ZiBo 255100, China)

e-mail: zhangzhendzq@163.com

\section{Jiaqun Wei}

Institution of Mathematics

School of Mathematics Science

Nanjing Normal University

Nanjing 210023, China

e-mail: weijiaqun@njnu.edu.cn 